\title{
BOUNDED FUNCTIONS STARLIKE WITH RESPECT TO SYMMETRICAL POINTS
}

FATIMA M. AL-OBOUDI

Department of Mathematics

Grrls College of Education

Sitteen Street, Malaz

Riyadh, SAUDI ARABIA

(Received September 28, 1994 and in revised form March 7, 1995)

ABSTRACT. Let $P[A, B],-1 \leq B<A \leq 1$, be the class of functions $p$ analytic in the unit disk $E$ with $p(0)=1$ and subordinate to $\frac{1+A z}{1+B z}$ In this paper we define and study the classes $S_{S}^{*}[A, B]$ of functions starlike with respect to symmetrical points A function $f$ analytic in $E$ and given by $f(z)=z+\sum_{n=2}^{\infty} a_{n} z^{n}$ is said to be in $S_{S}^{*}[A, B]$ if and only if, for $z \in E, \frac{2 z f^{\prime}(z)}{f(z)-f(-z)} \in P[A, B]$ Basic results on $S_{S}^{*}[A, B]$ are studied such as coefficient bounds, distortion and rotation theorems, the analogue of the Polya-Schoenberg conjecture and others

KEY WORDS AND PHRASES. Starlike functions with respect to symmetrical points, close-to-convex functions

1991 AMS SUBJECT CLASSIFICATION CODES. Primary 30C45

\section{INTRODUCTION}

Let $\mathcal{A}$ denote the class of functions, analytic in $E=\{z:|z|<1\}$ and normalized by the conditions $f(0)=0=f^{\prime}(0)-1 \quad$ In [7] Sakaguchi defined the class of starlike functions with respect to symmetrical points as follows

Let $f \in \mathcal{A}$ Then $f$ is said to be starlike with respect to symmetrical points in $E$ if, and only if,

$$
\operatorname{Re} \frac{z f^{\prime}(z)}{f(z)-f(-z)}>0, \quad z \in E \text {. }
$$

We denote this class by $S_{S}^{*}$ Obviously, it forms a subclass of close-to-convex functions and hence univalent Moreover, this class includes the class of convex functions and odd starlike functions with respect to the origin, see [7]

Janowski [4] introduced the classes $P[A, B]$ and $S^{*}[A, B]$ as follows

For $A$ and $B,-1 \leq B<A \leq 1$, a function $p$, analytic in $E$, with $p(0)=1$, belongs to the class $P[A, B]$ if $p(z)$ is subordinate to $\frac{1+A z}{1+B z}$

A function $f \in \mathcal{A}$ is said to be in $S^{*}[A, B]$, if and only if, $\frac{z f^{\prime}(z)}{f(z)} \in P[A, B]$

We now define the following

DEFINITION 1.1. Let $f \in \mathcal{A}$ Then $f \in S_{S}^{*}[A, B],-1 \leq B<A \leq 1$ if and only if, for $z \in E$

$$
\frac{2 z f^{\prime}(z)}{f(z)-f(-z)} \in P[A, B] \text {. }
$$


It is clear that $S_{S}^{*}[1,-1] \equiv S_{S}^{*}$ and $S_{S}^{*}[1-2 \alpha,-1] \equiv S_{S}^{*}(\alpha)$, the class of starlike functions with respect to symmetrical points of order $\alpha$ defined by Das and Singh [2]

To show that functions in $S_{S}^{*}[A, B]$ are univalent, we need the following

LEMA 1.1. [5] Let $p_{1}$ and $p_{2}$ belong to $P[A, B]$ and $\alpha, \beta$ any positive real numbers Then

$$
\frac{1}{\alpha+\beta}\left[\alpha p_{1}(a)+\beta P_{2}(z)\right] \in P[A, B] \text {. }
$$

THEOREM 1.1. Let $f \in S_{S}^{*}[A, B]$ Then the odd function

$$
\tau(z)=\frac{1}{2}[f(z)-f(-z)],
$$

belongs to $S^{*}[A, B]$

PROOF. Logarithmic differentiation of (1 3 ) gives

$$
\frac{z \tau^{\prime}(z)}{\tau(z)}=\frac{z f^{\prime}(z)}{f(z)-f(-z)}+\frac{z f^{\prime}(-z)}{f(z)-f(-z)}=\frac{1}{2}\left[p_{1}(z)+p_{2}(z)\right]
$$

where $p_{1}, p_{2} \in P[A, B]$, since $f \in S_{S}^{*}[A, B]$ Using Lemma 11 we have the required result

REMARK 1.1. From Theorem 11 and Definition 11 we conclude that

$$
S_{S}^{*}[A, B] \subset K,
$$

where $K$ is the class of close-to-convex functions This implies that functions in $S_{S}^{*}[A, B]$ are close-toconvex and hence univalent

\section{COEFFICIENT BOUNDS}

In the following we will study the coefficients problem for the class $S_{S}^{*}[A, B]$, we need the following

LEMMA 2.1 [1] Let $\tau$ be an odd function and $\tau \in S_{S}^{*}[1-2 \alpha,-1]$ and let $\tau(z)=$ $z+\sum_{n=2}^{\infty} b_{2 n-1} z^{2 n-1} \quad$ Then

$$
\left|b_{2 n-1}\right| \leq \frac{1}{(n-1) !} \prod_{v=0}^{n-2}[(1-\alpha)+v]
$$

This result is sharp as can be seen from the function

$$
\begin{aligned}
f_{o}(z) & =\frac{z}{\left(1-z^{2}\right)^{(1-\alpha)}} \\
& =z+\sum_{n=2}^{\infty}\left\{\frac{1}{(n-1) !}(1-\alpha)[(1-\alpha)+1] \ldots[(1-\alpha)+(n-2)]\right\} z^{2 n-1} .
\end{aligned}
$$

LEMMA 2.2. [1] Let $\tau$ be an odd function belonging to $S^{*}[A, B]$ and let $\tau(z)=z+\sum_{n=2}^{\infty}$ $b_{2 n-1} z^{2 n-1}$ Put $M=\left[\frac{A-B}{2(1+B)}\right]$, the largest integer not greater than $\frac{A-B}{2(1+B)}$. We have the following

(i) If $A-B>2(1+B)$, then

$$
\left|b_{2 n-1}\right| \leq \frac{1}{(n-1) !} \prod_{v=0}^{n-2}\left[\frac{A-B}{2}-v B\right], n=2,3, \ldots, M+1 \text {. }
$$

and

$$
\left|b_{2 n-1}\right| \leq \frac{1}{(n-1) M !} \prod_{v=0}^{M}\left[\frac{A-B}{2}-v B\right], n \geq M+2 .
$$


(ii) If $A-B \leq 2(1+B)$, then

$$
\left|b_{2 n} \quad\right| \mid \leq \frac{A-B}{2(n-1)}, n=1,2, \ldots
$$

The bounds in (2 1$)$ and (2 2) are sharp

LEMMA 2.3. [1] Let $p \in P[A, B]$ and $p(z)=1+\sum_{n=1}^{\infty} c_{n} z^{n}$.

Then

$$
\left|c_{n}\right| \leq A-B
$$

This result is sharp

To solve the coefficient problem for the class $S_{S}^{*}[1-2 \alpha,-1]$ we will use the technique of dominant power series which is defined as follows

Let $f$ and $F$ be given by the power series

$$
f(z)=\sum_{n=0}^{\infty} a_{n} z^{n} \quad \text { and } \quad F(z)=\sum_{n=0}^{\infty} A_{n} z^{n},
$$

convergent in some disk $E_{R}:|z|<R, R>0$ We say that $f$ is dominated by $F$ (or $F$ dominates $f$ ), and we write $f \ll F$ if for each integer $n \geq 0$

$$
\left|a_{n}\right| \leq A_{n} .
$$

THEOREM 2.1. Let $f \in S_{S}^{*}[1-2 \alpha,-1]$ and be given by $f(z)=z+\sum_{n=2}^{\infty} a_{n} z^{n}$. Then

(i) $\left|a_{2}\right| \leq(1-\alpha),\left|a_{3}\right| \leq(1-\alpha)$.

(ii) $\left|a_{2 n}\right| \leq \frac{(1-\alpha)}{n}\left\{1+\sum_{k=2}^{n}\left[\frac{1}{(k-1) !} \prod_{v=0}^{k-2}((1-\alpha)+v)\right]\right\}, n \geq 2$.

(iii) $\left|a_{2 n-1}\right| \leq \frac{2(1-\alpha)}{2 n-1}\left\{1+\sum_{k=2}^{n-1}\left[\frac{1}{(k-1) !} \prod_{v=0}^{k-2}((1-\alpha)+v)\right]\right\}$

$$
+\frac{!}{(2 n-1)(n-1) !} \prod_{v=0}^{n-2}((1-\alpha)+v), n \geq 3 \text {. }
$$

These bounds are sharp.

PROOF. Since $f \in S_{S}^{*}[1-2 \alpha,-1]$, then by Theorem 1.1 (with $A=1-2 \alpha, B=-1$ ) there exists an odd starlike function of order $\alpha, \tau$ where $\tau(z)=\frac{1}{2}[f(z)-f(-z)]$ such that

$$
z f^{\prime}(z)=\tau(z) p(z), \quad p \in P[1-2 \alpha,-1] .
$$

From Lemma 2.1 we see that

$$
\tau(z) \ll \frac{z}{\left(z-z^{2}\right)^{(1-\alpha)}}
$$

and it is known [1] that

$$
p(z) \ll \frac{1+(1-2 \alpha) z}{(1-z)} .
$$

Hence using these facts with (2.3) we obtain

$$
z f^{\prime}(z) \ll\left[\frac{z}{\left(1-z^{2}\right)^{(1-\alpha)}} \cdot \frac{1+(1-2 \alpha) z}{(1-z)}\right] .
$$

Simple calculations show that 


$$
\frac{z(1+(1-2 \alpha) z)}{\left.(1-z)\left(1-z^{2}\right)^{(1} \alpha\right)}=z+\sum_{n=2}^{\infty} A_{n} z^{n}
$$

where

$$
\begin{aligned}
A_{2}= & 2(1-\alpha), A_{3}=3(1-\alpha) \\
A_{2 n}= & 2(1-\alpha)\left\{1+\sum_{k=2}^{n}\left[\frac{1}{(k-1) !} \prod_{v}^{k}{ }_{0}^{2}((1-\alpha)+v)\right]\right\}, n \geq 2 \\
A_{2 n-1}= & 2(1-\alpha)\left\{1+\sum_{k=2}^{n-1}\left[\frac{1}{(k-1) !} \prod_{v=0}^{k-2}((1-\alpha)+v)\right]\right\} \\
& +\frac{1}{(n-1) !} \prod_{v=0}^{n-2}((1-\alpha)+v), n \geq 3 .
\end{aligned}
$$

Using this in (2 3) we obtain the required result

These bounds are sharp as can be seen from the function

$$
f(z)=\int_{0}^{z} \frac{(1+(1-2 \alpha) \xi)}{(1-\xi)\left(1-\xi^{2}\right)^{(1-\alpha)}} d \xi \in S_{S}^{*}[1-2 \alpha,-1] .
$$

The method of proof used in the above theorem unfortunately does not work for the general class $S_{S}^{*}[A, B]$ However, the above coefficients bounds for $S_{S}^{*}[1-2 \alpha,-1]$ do suggest the form of coefficients bounds for functions in $S_{S}^{*}[A, B]$ In fact we have the following.

THEOREM 2.2. Let $f \in S_{S}^{*}[A, B]$ and be given by $f(z)=z+\sum_{n=2}^{\infty} a_{n} z^{n} \quad$ Let $M$ be as in Lemma 22 Then we have the following

(i) $\left|a_{2}\right| \leq \frac{A-B}{2},\left|a_{3}\right| \leq \frac{A-B}{2}$

(ii) If $A-B>2(1+B)$, then for $n=2,3, \ldots, M+1$

$$
\left|a_{2 n}\right| \leq \frac{A-B}{2 n}\left\{1+\sum_{k=2}^{n}\left[\frac{1}{(k-1) !} \prod_{v=0}^{k-2}\left(\frac{A-B}{2}-v B\right)\right]\right\}
$$

and for $n=3,4, \ldots, M+1$

$$
\begin{aligned}
\left|a_{2 n-1}\right| & \leq \frac{A-B}{2 n-1}\left\{1+\sum_{k=2}^{n-1}\left[\frac{1}{(k-1) !} \prod_{v=0}^{k-2}\left(\frac{A-B}{2}-v B\right)\right]\right\} \\
& +\frac{1}{(2 n-1)(n-1) !} \prod_{v=0}^{n-2}\left(\frac{A-B}{2}-v B\right) .
\end{aligned}
$$

and for $n \geq M+2$

$$
\left|a_{2 n}\right| \leq \frac{A-B}{2 n}\left\{1+\sum_{k=2}^{n}\left[\frac{1}{(k-1) M !} \prod_{v=0}^{M}\left(\frac{A-B}{2}-v B\right)\right]\right\}
$$

and

$$
\begin{aligned}
\left|a_{2 n-1}\right| & \leq \frac{A-B}{2 n-1}\left\{1+\sum_{k=2}^{n-1}\left[\frac{1}{(k-1) M !} \prod_{v=0}^{M}\left(\frac{A-B}{2}-v B\right)\right]\right\} \\
& +\frac{1}{(2 n-1)(n-1) M !} \prod_{v=0}^{M}\left(\frac{A-B}{2}-v B\right) .
\end{aligned}
$$

(iii) If $A-B \leq 2(1+B)$, then 
and

$$
\left|a_{2 n}\right| \leq \frac{A-B}{2 n}\left\{1+\sum_{k=2}^{n} \frac{A-B}{2(k-1)}\right\}, n=2,3,
$$

$$
\left.\left|a_{2 n-1}\right| \leq \frac{A-B}{2 n-1}\left\{1+\sum_{k=2}^{n-1} \frac{A-B}{2(k-1)}+\frac{1}{2(n-1)}\right\}, n=3,4, \ldots\right\}
$$

The bounds in (2 5), (2 6) and (2.7) are sharp

PROOF. Since $f \in S_{S}^{*}[A, B]$, then by Theorem 21 there exists an odd function $\tau \in S^{*}[A, B]$ where $\tau(z)=\frac{1}{2}[f(z)-f(-z)]$ such that

$$
z f^{\prime}(z)=\tau(z) p(z), p \in P[A, B] .
$$

Let $\tau(z)=z+\sum_{n=2}^{\infty} b_{2 n-1} z^{2 n-1}$ and $p(z)=1+\sum_{n=1}^{\infty} c_{n} z^{n}$

Then

$$
z+\sum_{n=2}^{\infty} n a_{n} z^{n}=\left[z+\sum_{n=2}^{\infty} b_{2 n-1} z^{2 n-1}\right]\left[1+\sum_{n=1}^{\infty} c_{n} z^{n}\right] .
$$

Equating the coefficients of $z^{2}, z^{3}, z^{2 n}$ and $z^{2 n-1}$ in both sides we obtain

$$
\begin{gathered}
2 a_{2}=c_{1} \\
3 a_{3}=c_{2}+b_{3}, \\
2 n a_{2 n}=c_{2 n-1}+\sum_{k=2}^{n} b_{2 k-1} c_{2 n-(2 k-1)} \\
(2 n-1) a_{2 n-1}=c_{2 n-2}+\sum_{k=2}^{n-1} b_{2 k-1} c_{2 n-2 k}+b_{2 n-1} .
\end{gathered}
$$

Hence

$$
\begin{gathered}
\left|a_{2}\right| \leq \frac{\left|c_{1}\right|}{2} \\
\left|a_{3}\right| \leq \frac{\left|c_{2}\right|}{3}+\frac{\left|b_{3}\right|}{3} \\
2 n\left|a_{2 n}\right| \leq\left|c_{2 n-1}\right|+\sum_{k=2}^{n}\left|b_{2 k-1}\right|\left|c_{2 n-(2 k-1)}\right|
\end{gathered}
$$

and

$$
(2 n-1)\left|a_{2 n-1}\right| \leq\left|c_{2 n-2}\right|+\sum_{k=2}^{n-1}\left|b_{2 k-1}\right|\left|c_{2 n-2 k}\right|+\left|b_{2 n-1}\right| .
$$

Using Lemma 23 we obtain

$$
\begin{gathered}
\left|a_{2}\right| \leq \frac{A-B}{2},\left|a_{3}\right| \leq \frac{A-B}{6}+\frac{\left|b_{3}\right|}{3} \\
\left|a_{2 n}\right| \leq \frac{A-B}{2 n}\left\{1+\sum_{k=2}^{n}\left|b_{2 k-1}\right|\right\}, n \geq 2
\end{gathered}
$$


and

$$
\left|a_{2 n} \quad\right|\left|\leq \frac{A-B}{2 n-1}\left\{1+\sum_{k=2}^{n-1}\left|b_{2 k-1}\right|\right\}+\frac{1}{2 n-1}\right| b_{2 n-1} \mid, \quad n \geq 3 .
$$

Using Lemma 22 we get the required result The bounds in (2 5) and (26) are sharp as can be seen from the function

$$
f(z)= \begin{cases}\int_{0}^{z}\left(\frac{1-A \xi^{n}}{1-B \xi^{n}}\right)\left(1+B \xi^{2}\right)^{\frac{A-B}{2 B}} d \xi, & B \neq 0 \\ \int_{0}^{z}\left(1-A \xi^{n}\right) \exp \left(A \xi^{2} / 2\right) / \xi d \xi, & B=0 .\end{cases}
$$

While the bounds in (2 7) are sharp as can be seen from the function

$$
f(z)=\int_{0}^{z} \frac{1-A \xi^{n}}{1-B \xi^{n}} \exp \left[\frac{A-B}{2 n} \xi^{2 n}\right] d \xi .
$$

SPECIAL CASE. For $a=1, B=-1$ we see that

$$
\left|a_{n}\right| \leq 1, \quad n \geq 2,
$$

which is the coefficient bounds for the class $S_{S}^{*}$ obtained by Sakaguchi [7].

\section{DISTORTION AND ROTATION THEOREMS}

To derive our results we need the following

LEMMA 3.1. [3] Let $f \in S^{*}[A, B]$. Then for $|z|=r<1$

$$
\begin{array}{ll}
r(1-B r)^{\frac{A-B}{B}} \leq|f(z)| \leq r(1+B r)^{\frac{A-B}{B}} & \text { for } \quad B \neq 0 \\
r \exp (-A r) \leq|f(z)| \leq r \exp (A r) & \text { for } \quad B=0 .
\end{array}
$$

These bounds are sharp.

LEMMA 3.2. [4] Let $p \in P[A, B]$, then for $|z|=r<1$

$$
\frac{1-A r}{1-B r} \leq \operatorname{Rep}(z) \leq|p(z)| \leq \frac{1+A r}{1+B r} .
$$

These bounds are sharp.

THEOREM 3.1. Let $f \in S_{S}^{*}[A, B]$. Then for $|z|=r<1$.

(i)

$$
\left(\frac{1-A r}{1-B r}\right)\left(1-B r^{2}\right)^{\frac{A-B}{2 B}} \leq\left|f^{\prime}(z)\right| \leq\left(\frac{1+A r}{1+B r}\right)\left(1+B r^{2}\right)^{\frac{A-B}{2 B}}, \quad B \neq 0
$$

and

$$
\begin{gathered}
(1-A r) \exp \left(-\frac{A r^{2}}{2}\right) \leq\left|f^{\prime}(z)\right| \leq(1+A r) \exp \left(\frac{A r^{2}}{2}\right), \quad B=0 \\
\text { (ii) } \int_{0}^{r}\left(\frac{1-A r}{1-B r}\right)\left(1-B r^{2}\right)^{\frac{A-B}{2 B}} d r \leq|f(z)| \leq \int_{0}^{r}\left(\frac{1+A r}{1+B r}\right)\left(1+B r^{2}\right)^{\frac{A-B}{2 B}} d r, \quad B \neq 0 \\
\int_{0}^{r}(1-A r) \exp \left(\frac{-A r^{2}}{2}\right) d r \leq|f(z)| \leq \int_{0}^{r}(1+A r) \exp \left(\frac{A r^{2}}{2}\right) d r, \quad B=0
\end{gathered}
$$

These bounds are sharp

PROOF. Since $f \in S_{S}^{*}[A, B]$, then from (2.8) we have 


$$
\left|z f^{\prime}(z)\right|=|p(z)||\tau(z)|
$$

where $p \in P[A, B]$ and $\tau(z)=\frac{1}{2}[f(z)-f(-z)]$ and $\tau \in S^{*}[A, B]$ (Theorem 11 )

Using Lemma 31 , we have the following bounds for the distortion of the odd function $\tau \in S^{*}[A, B]$ for $|z|=r<1$,

$$
r\left(1-B r^{2}\right)^{\frac{A-B}{2 B}} \leq|\tau(z)| \leq r\left(1-B r^{2}\right)^{\frac{A-B}{2 B}}, \quad B \neq 0
$$

and

$$
r \exp \left(-\frac{A r^{2}}{2}\right) \leq|\tau(z)| \leq r \exp \left(\frac{A r^{2}}{2}\right), \quad B=0 .
$$

Using Lemma 32 and (36) in ( 35 ) we obtain the required result

Equality signs in (3 1), (3 2), (3 3 ) and (3 4) are attained by the function $f_{*} \in S_{S}^{*}[A, B]$ given by

$$
f_{*}^{\prime}(z)= \begin{cases}\left(\frac{1+A \delta_{1} z}{1+B \delta_{1} z}\right)\left(1+B \delta_{2} z^{2}\right)^{\frac{1-B}{2 B}}, & B \neq 0 \\ \left(1+\delta_{1} A z\right) \exp \left(\frac{A \delta_{2} z^{2}}{2}\right), & B=0,\left|\delta_{1}\right|=\left|\delta_{2}\right|=1\end{cases}
$$

SPECIAL CASE. For $A=1-2 \alpha, B=-1$, we get the distortion theorems for $f \in S_{S}^{*}(\alpha)$, see [2]

Before proving the rotation theorem for $f \in S_{S}^{*}[A, B]$, we need the following

LEMMA 3.3. [3] Let $g \in S^{*}[A, B]$ Then for $|z|=r<1$

$$
\left|\arg \frac{g(z)}{z}\right| \leq\left\{\begin{array}{l}
\frac{A-B}{B} \sin ^{-1}(B r), \quad B \neq 0 \\
A r, \quad B=0
\end{array}\right\}
$$

These bounds are sharp

THEOREM 3.2. Let $f \in S_{S}^{*}[A, B]$. Then for $|z|=r<1$

$$
\left|\arg f^{\prime}(z)\right| \leq\left\{\begin{array}{l}
\frac{A-B}{2 B} \sin ^{-1}\left(B r^{2}\right)+\sin ^{-1} \frac{(A-B) r}{1-A B r^{2}}, \quad B \neq 0 \\
\frac{A r^{2}}{2}+\sin ^{-1}(A r), \quad B=0
\end{array}\right.
$$

These bounds are sharp.

PROOF. From (2.8) we have

$$
\left|\arg f^{\prime}(z)\right| \leq\left|\arg \frac{\tau(z)}{z}\right|+|\arg p(z)|,
$$

where $\tau$ is an odd function $\tau \in S^{*}[A, B]$ and $\tau(z)=\frac{1}{2}[f(z)-f(-z)], p \in P[A, B]$. It is known [4] that for $p \in P[A, B]$ and for $|z|=r<1$

$$
\left|p(z)-\frac{1-A B r^{2}}{1-B^{2} r^{2}}\right| \leq \frac{(A-B) r}{1-B^{2} r^{2}}
$$

from which it follows that

$$
|\arg p(z)| \leq \sin ^{-1} \frac{(A-B) r}{1-A B r^{2}} .
$$

Using Lemma 3.3, we have the following bounds for the argument of the odd function $\tau \in S^{*}[A, B]$ (notice that $\tau(z)=\sqrt{g\left(z^{2}\right)}$ ) 
Using Lemma 33 , we have the following bounds for the argument of the odd function $\tau \in S^{*}[A, B]$ (notice that $\tau(z)=\sqrt{g\left(z^{2}\right)}$ )

$$
\left|\arg \frac{\tau(z)}{z}\right| \leq\left\{\begin{array}{l}
\frac{A-B}{2 B} \sin ^{1}\left(B r^{2}\right), \quad B \neq 0 \\
\frac{A r^{2}}{2}, \quad B=0
\end{array} .\right.
$$

Using (3 9) and (3 10) in (3 8) we get the required result

Equality signs are attained by the function $f_{*} \in S_{S}^{*}[A, B]$ given by (3 7)

\section{THE ANALOGUE OF THE POLYA-SCHOENBERG CONJECTURE}

In 1973 Ruscheweyh and Sheil-Small [6] proved the Polya-Schoenberg conjecture namely if $f$ is convex or starlike or close-to-convex and $\phi$ is convex, then $f * \phi$ belongs to the same class, where (*) stands for Hadamard product or convolution In the following we shall prove the analogue of this conjecture for the class $S_{S}^{*}[A, B]$ and give some of its applications We need the following

LEMMA 4.1. [6] Let $\phi$ be convex and $g$ starlike Then for $F$ analytic in $E$ with $F(0)=1$, $\frac{\phi * F g}{\phi * g}(E)$ is contained in the convex hull of $F(E)$

THEOREM 4.1. Let $f \in S_{S}^{*}[A, B]$ and let $\phi$ be convex Then $(f * \phi) \in S_{S}^{*}[A, B]$

PROOF. To prove that $(f * \phi) \in S_{S}^{*}[A, B]$, it is sufficient to show that $\frac{2 z(f * \phi)^{\prime}(z)}{(f * \phi)(z)-(f * \phi)(-z)}$ is contained in the convex hull of $\frac{2 z f^{\prime}(z)}{f(z)-f(-z)}$

Now

$$
\begin{aligned}
\frac{2 z(f * \phi)^{\prime}(z)}{(f * \phi)-(f * \phi)(-z)} & =\frac{2 z f^{\prime}(z) * \phi(z)}{[f(z)-f(-z)] * \phi(z)} \\
& =\frac{\phi(z) * \frac{2 z f^{\prime}(z)}{f(z)-f(-z)} \cdot \frac{f(z)-f(-z)}{2}}{\phi(z) * \frac{f(z)-f(-z)}{2}}
\end{aligned}
$$

Applying Lemma 41, with $g(z)=\frac{[f(z)-f(-z)]}{2} \in S^{*}[A, B]$ and $F(z)=\frac{2 z f^{\prime}(z)}{f(z)-f(-z)}$, we obtain the required results

REMARKS 4.1. As an application of Theorem 41 we note that the family $S_{S}^{*}[A, B]$ is invariant under the following operators

$$
\begin{aligned}
F_{1}(f) & =\int_{0}^{z} \frac{f(\xi)}{\xi} d \xi=\left(f * \phi_{1}\right)(z) \\
F_{2}(f) & =\frac{2}{z} \int_{0}^{z} f(\xi) d \xi=\left(f * \phi_{2}\right)(z) \\
F_{3}(f) & =\int_{0}^{z} \frac{f(\zeta)-f(x \zeta)}{\xi-x \zeta} d \zeta,|x| \leq 1, x \neq 1 \\
& =\left(f * \phi_{3}\right)(z) \\
F_{4}(f) & =\frac{1+c}{c} \int_{0}^{z} \xi^{c-1} f(\xi) d \xi, \quad \operatorname{Re} c>0 \\
& =\left(f * \phi_{4}\right)(z)
\end{aligned}
$$

where $\phi_{2}(i=1,2,3,4)$ are convex, and 


$$
\begin{aligned}
& \phi_{1}(z)=\sum_{n-1}^{\infty} \frac{1}{n} z^{n}=-\log (1-z), \\
& \phi_{2}(z)=\sum_{n=1}^{\infty} \frac{2}{n+1} z^{n}=\frac{-2[z+\log (1-z)]}{z}, \\
& \phi_{3}(z)=\sum_{n=1}^{\infty} \frac{1-x^{n}}{n(1-x)} z^{n}=\frac{1}{1-x} \log \frac{1-x z}{1-z},|x| \leq 1, x \neq 1, \\
& \phi_{4}(z)=\sum_{n=1}^{\infty} \frac{1+c}{n+c} z^{n}, \operatorname{Re} c>0 .
\end{aligned}
$$

\section{REFERENCES}

[1] ANH, V , K fold symmetric starlike univalent functions, Bull. Austral. Math. Soc. 32 (1985), 419436

[2] DAS, R and SINGH, P, On properties of certain subclasses of close-to-convex functions, Ann. Univ. Marıae Curıe Sklodowska 2 (1976), 15-22.

[3] GOHL, R and MEHROK, B, On a class of close-to-convex functions, Indian J. Pure Appl. Math. $12(5)(1981), 648-658$

[4] JANOWSKI, W, Some extremal problem for certain families of analytic functions I, Ann. Polon. Math. 28 (1973), 298-326

[5] NOOR, K, On some univalent integral operators, J. of Math. Anal. and Appl. 2 (1987), 586-592

[6] RUSCHEWEYH, S. and SHEIL-SMALL, T., Hadamard products of Schlicht functions and the Polya-Schoenberg conjecture, Comment. Math. Helv. 48 (1979), 119-135.

[7] SAKAGUCHI, K., On certain univalent mapping, J. Math. Soc. Japan, 11 (1959), $72-75$. 


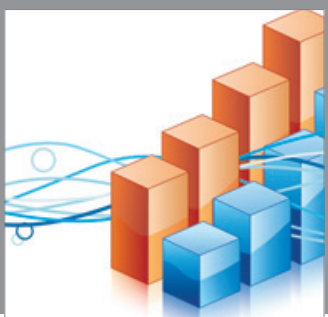

Advances in

Operations Research

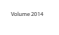

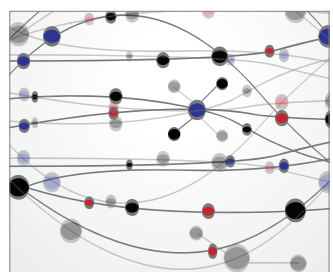

\section{The Scientific} World Journal
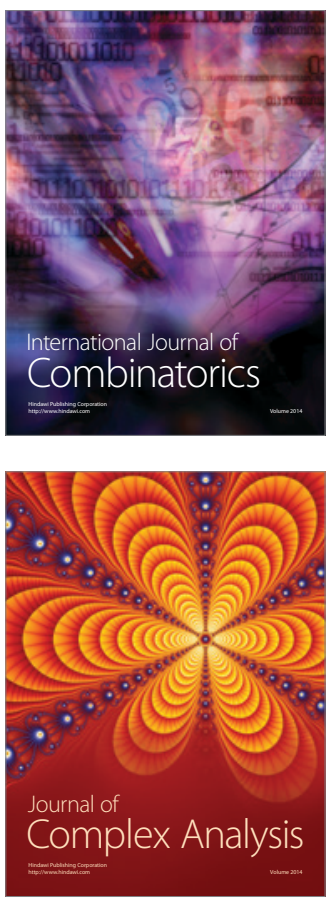

International Journal of

Mathematics and

Mathematical

Sciences
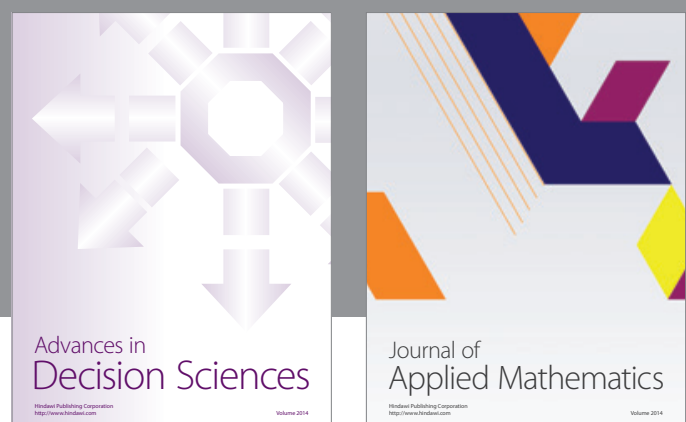

Journal of

Applied Mathematics
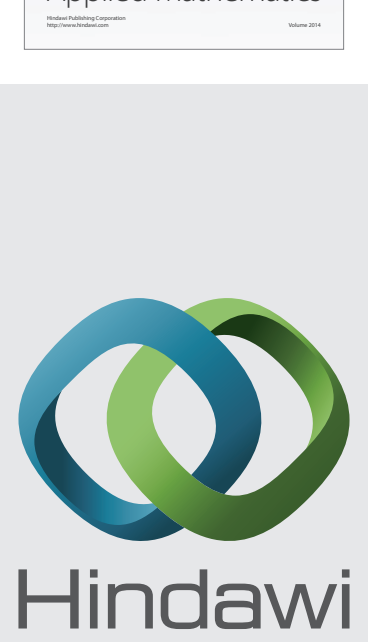

Submit your manuscripts at http://www.hindawi.com
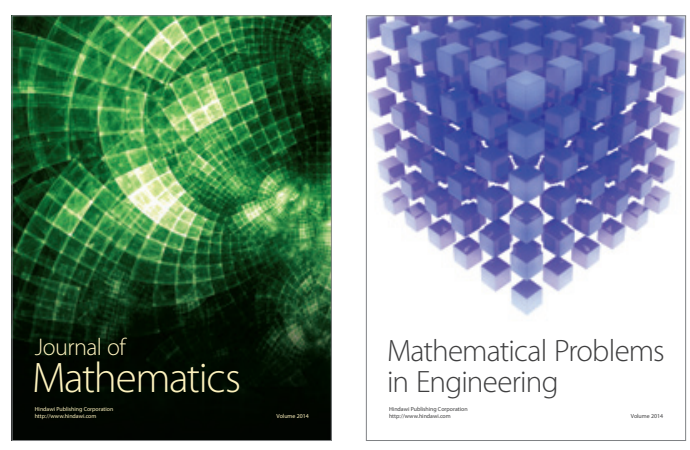

Mathematical Problems in Engineering
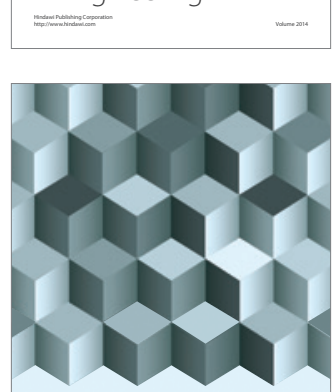

Journal of

Function Spaces
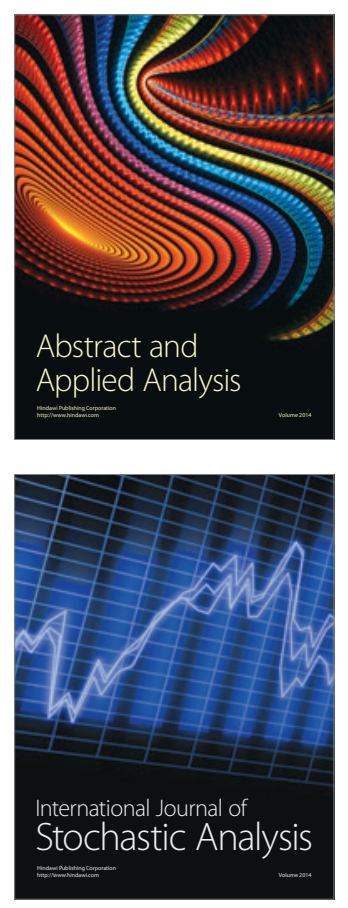

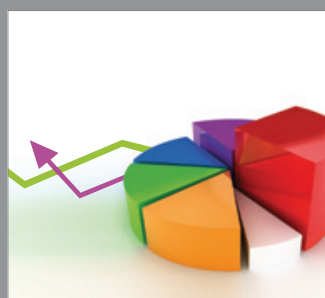

ournal of

Probability and Statistics

Promensencen
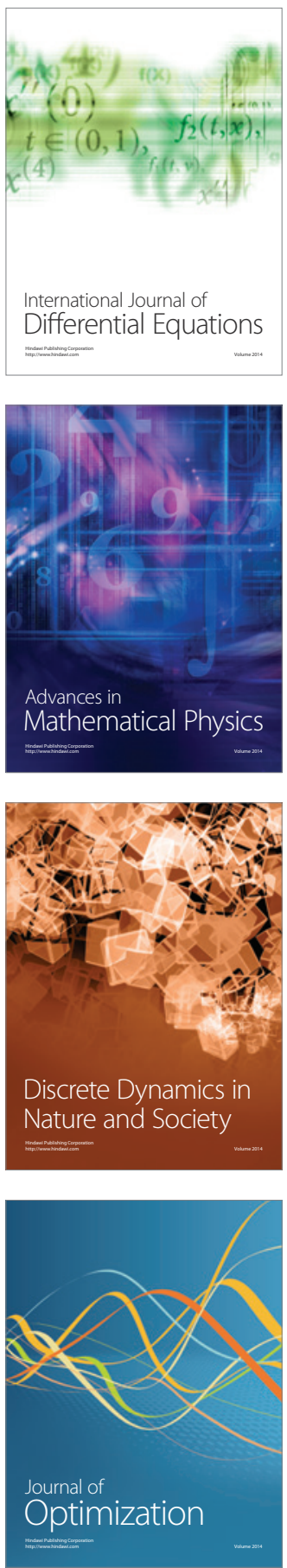